Pulsar Astronomy - 2000 and Beyond

ASP Conference Series, Vol. 202, 2000

M. Kramer, N. Wex, and R. Wielebinski, eds.

\title{
Applications of Ohm's Law to the pulsar magnetosphere
}

\author{
Thomas Kunzl ${ }^{1,2}$,Harald Lesch ${ }^{1}$ and Axel Jessner ${ }^{3}$ \\ (1) Institut für Astronomie und Astrophysik der Universität München, \\ Scheinerstr. 1, D-81679 München \\ (2) Max-Planck-Institut für extraterrestrische Physik, Gießenbachstr., \\ D-85748 Garching \\ (3) Max-Planck- Institut für Radioastronomie, Auf dem Hügel 69, \\ D-53121 Bonn
}

\begin{abstract}
We show that in the inner magnetosphere of a pulsar mildly relativistic particles can flow out steadily under two assumptions: i) all vacuum fields are completely shielded by copious amounts of particles drawn out by thermal and field emission (which is likely as shown in Jessner et al., 1999) ii) particles emitted from the neutron star surface have weakly relativistic energies $(\beta \gamma \approx 1)$. The results are consistent with the typical particle energies predicted in the radio emission model by Kunzl et al., 1999.
\end{abstract}

\section{Description of the model}

Imagine a hot neutron star that constantly emits enough charged particles to shield the induced electric acceleration fields. In such cases only non-idealities in the outflowing plasma can lead to accelerating fields. Since there are no external forces in this case we can apply Ohm's law. Here we use a more general form than can normally be found and include also time variations of the current and gradients in the distribution function.

$$
\frac{\partial \vec{j}}{\partial t}+\operatorname{grad}(\vec{v} \vec{j}+\vec{j} \vec{v}-\vec{v} \vec{v} \rho)=\frac{\rho e}{m_{\mathrm{e}}} \vec{E}+\frac{\rho e}{m_{\mathrm{e}} c} \vec{v} \times \vec{B}+\rho \int \vec{v} \frac{\mathrm{d} f(\vec{v})}{\mathrm{d} t} \mathrm{~d} \vec{v}
$$

This equation is valid for strong magnetic fields (= no pressure term, no currents perpendicular to the magnetic field). $\rho$ is the (electron) charge density, the distribution function is normalized in that way that $\int f(\vec{v}) \mathrm{d}^{3} v=1$. The other symbols have their usual meaning. Besides, we use the following simplifying assumptions: $\vec{v} \| \vec{j}$, namely $\vec{j}=\rho \vec{v}$ which is automatically fulfilled for a pure electron flow and $\frac{\mathrm{d}}{\mathrm{d} t} f(\vec{v})=0$ (collisionless plasma). The first term of $(1)$ is fluctuating rapidly (approx. with $\omega_{\text {pe }}$ ) and will therefore not play an important role apart from some resonant particles. The third term $(\vec{j} \times \vec{B} \perp \vec{B})$ does not contibute to the parallel (=accelerating) field and is therefore neglected as well.

Evaluating the remaining equation for the dipolar geometry and neglecting all terms proportional to $v_{\theta}$ we can normalize to dimensionless units (accelerating 
force $\left.\rightarrow \frac{\partial}{\partial t}(\beta \gamma):=\frac{\partial}{\partial t} \varphi\right)$. This yields:

$$
\frac{\mathrm{d} \varphi}{\mathrm{d} x}=\beta_{\|}^{2} \frac{\partial}{\partial x_{\|}} \ln \frac{\rho}{\rho_{\mathrm{GJ}}}+\beta_{\perp} \beta_{\|} \frac{\partial}{\partial x_{\phi}} \ln \frac{\rho}{\rho_{\mathrm{GJ}}}+\beta_{\perp} \frac{\partial}{\partial x_{\phi}} \beta_{\|}
$$

where $\varphi=\beta \gamma$. The second and third term only play a role in the outer magnetosphere, so for processes inside the radio emission zone we can neglect them (they are about a factor of $r / r_{L C}$ smaller than the first term).

\section{Results}

Integrating equation (2) numerically for the classical Goldreich-Julian

energy gain of particles up to xem vs. initial energy

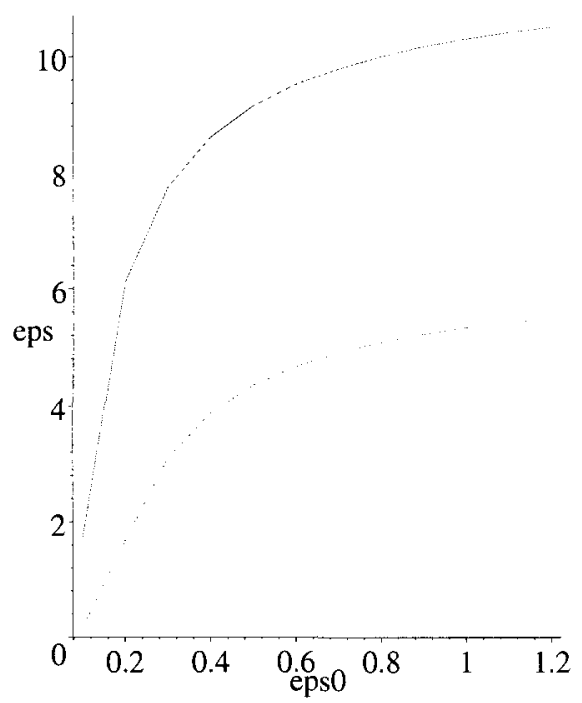

Figure 1: Dimensionless energy gain of electrons by the calculated fields for normal (lower) and millisecond pulsars (upper) versus the initial kinetic energy (in units of $\beta \gamma$ ). The parameters for this example are $P=$ $0.5 \mathrm{~s}$, emission height $x_{\mathrm{em}}=50$ (normal pulsar) and $P=5 \mathrm{~ms}, x_{\mathrm{em}}=8$ (ms pulsar), respectively. In both cases we took a fieldline that crosses the surface at $\theta_{\text {cap }}$. solution we find that when we start with low relativistic particles $(\phi \approx 1)$ up to a typical emission height (50 pulsar radii for normal and 8 for mspulsars, e.g. Kijak and Gil, 1998; Blaskiewicz et al., 1991) the particles are accelerated up to Lorentz factors of about $10-15$ which is close to the particle energies needed for the most intense (low frequency) radio emission in the Kunzl et al., 1999 model.

\section{References}

Blaskiewicz, M., Cordes, J.M., Wasserman, I., 1991, ApJ, 370,643

von Hoensbroech, A., Lesch, H., Kunzl, T., 1998, A\&A, 336, 209

Jessner, A., Lesch, H., Kunzl, T., 1999 , in prep.

Kijak J., Gil, J.A., 1998, MNRAS, 288,631

Kunzl, T.,Lesch, H., Jessner, A., von Hoensbroech, A., 1999, ApJ, 505, L 139

Kunzl, T., Lesch, H., Jessner, A., 1999, submitted to ApJ

Lesch, H., Jessner, A., Kramer, M., Kunzl,T., 1998, A\&A, 332, L21

Muslimov, A.G., Tsygan, A.I., 1992, MNRAS, 255, 61

Ruderman, M.A., Sutherland, P.G., 1975, ApJ, 196, 51

Sturrock, P.A., 1971, ApJ, 164, 529 